\title{
Germline PRKACA amplification causes variable phenotypes that may depend on the extent of the genomic defect: molecular mechanisms and clinical presentations
}

\author{
Maya B Lodish, Bo Yuan ${ }^{1}$, Isaac Levy, Glenn D Braunstein², Charalampos Lyssikatos, \\ Paraskevi Salpea, Eva Szarek, Alexander S Karageorgiadis, Elena Belyavskaya, \\ Margarita Raygada, Fabio Rueda Faucz, Louise Izatt ${ }^{3}$, Caroline Brain4, \\ James Gardner ${ }^{5}$, Martha Quezado6 ${ }^{6}$ J Aidan Carney, James R Lupski ${ }^{1,8,9}$ and \\ Constantine A Stratakis
}

Section on Endocrinology and Genetics, Program on Developmental Endocrinology and Genetics, National Institute of Child Health and Human Development, National Institutes of Health, Building 10-CRC, Room 9D42, 10 Center Drive, MSC, 1103, Bethesda, Maryland 20892, USA, 'Department of Molecular and Human Genetics, Baylor College of Medicine, Houston, Texas, USA, ${ }^{2}$ Department of Medicine, Cedars-Sinai Medical Center, Los Angeles, California, USA, ${ }^{3}$ Department of Clinical Genetics, Guy's Hospital, London, UK, ${ }^{4}$ Department of Endocrinology, Great Ormond Street Hospital for Children, London, UK, ${ }^{5}$ Department of Pediatrics, Our Lady of the Lake Children's Hospital, Baton Rouge, Louisiana, USA, ${ }^{6}$ Department of Pathology, National Cancer Institute, Bethesda, Maryland, USA, ${ }^{7}$ Emeritus Member, Department of Laboratory Medicine and Pathology, Mayo Clinic, Rochester, Minnesota, USA, ${ }^{8}$ Department of Pediatrics, Baylor College of Medicine, Houston, Texas, USA and ${ }^{9}$ Texas Children's Hospital, Houston, Texas, USA

Correspondence should be addressed to M B Lodish

Email lodishma@mail.nih.gov

\begin{abstract}
Objective: We have recently reported five patients with bilateral adrenocortical hyperplasia (BAH) and Cushing's syndrome (CS) caused by constitutive activation of the catalytic subunit of protein kinase A (PRKACA). By doing new in-depth analysis of their cytogenetic abnormality, we attempted a better genotype-phenotype correlation of their PRKACA amplification.

Design: This study is a case series.

Methods: Molecular cytogenetic, genomic, clinical, and histopathological analyses were performed in five patients with CS. Results: Reinvestigation of the defects of previously described patients by state-of-the-art molecular cytogenetics showed complex genomic rearrangements in the chromosome 19p13.2p13.12 locus, resulting in copy number gains encompassing the entire PRKACA gene; three patients (one sporadic case and two related cases) were observed with gains consistent with duplications, while two sporadic patients were observed with gains consistent with triplications. Although all five patients presented with ACTH-independent CS, the three sporadic patients had micronodular BAH and underwent bilateral adrenalectomy in early childhood, whereas the two related patients, a mother and a son, presented with macronodular BAH as adults. In at least one patient, PRKACA triplication was associated with a more severe phenotype.

Conclusions: Constitutional chromosomal PRKACA gene amplification is a recently identified genetic defect associated with $C S$, a trait that may be inherited in an autosomal dominant manner or occur de novo. Genomic rearrangements can be complex and can result in different copy number states of dosage-sensitive genes, e.g., duplication and triplication. PRKACA amplification can lead to variable phenotypes clinically and pathologically, both micro- and macro-nodular BAH, the latter of which we speculate may depend on the extent of amplification.
\end{abstract}




\section{Introduction}

Inactivating mutations of PRKAR1A, the main regulatory subunit $(\mathrm{R} 1 \alpha)$ of protein kinase A (PKA), cause primary pigmented nodular adrenocortical disease (PPNAD), a bilateral adrenocortical hyperplasia (BAH) leading to Cushing's syndrome (CS) (1). PRKAR1A maps to 17q24.2 and is frequently deleted in patients with PPNAD with and without Carney complex (CNC), as well as in sporadic patients with cortisol-secreting adrenal adenomas $(2,3,4)$. Inactivation of PRKAR1A leads to increased PKA activity that is due to lack of regulation of the PKA's main catalytic subunit $(\mathrm{C} \alpha$ ) coded by the PRKACA gene (3).

We have recently reported gain-of-function mutations in PRKACA that lead to increased cAMP signaling in adrenal CS associated with BAH (when PRKACA defects are present in the germline) and sporadic cortisolproducing adrenal adenomas (CPAs) (when PRKACA defects are present in the somatic state, i.e., somatic events confined to the tumor tissue) (4). Thus, gain of function of PRKACA appears to lead to benign adrenal tumors without any other manifestations of CNC (such as skin pigmentation, myxomas, and other neoplasms), suggesting that increased activity of this catalytic subunit of PKA is associated with abnormalities specific to adrenocortical tissue, whereas inactivating mutations of the PKA regulatory subunit $\mathrm{R} 1 \alpha$ (encoded by PRKAR1A) result in the full spectrum of findings associated with CNC.

All patients had been sequenced for PRKAR1A, PRKACA and PRKACB and their sequences were normal (4). In the present investigation, using in-depth probes, we investigated the nature of the cytogenetic defects in all patients with germline PRKACA amplification. We also collected additional phenotypic information on the probands and their families. Surprisingly, our new data suggest that there might be a dosage-dependent effect on the phenotype, as both duplications and triplications encompassing PRKACA were observed, and those patients with triplications presented at a younger age. In addition, one patient developed breast cancer that was found to express PRKACA. The type of $\mathrm{BAH}$ varied from PPNAD, to non-pigmented isolated micronodular adrenocortical disease (iMAD) and macronodular adrenocortical disease also known as primary macronodular adrenocortical hyperplasia (PMAH).

\section{Subjects and methods}

\section{Patients}

The Institutional Review Boards of all the participating institutions approved the investigation. Written informed consent was obtained from all patients and/or their guardians. In our previous report, the details of the clinical presentation of the patients with germline amplification of the PRKACA gene were not included (4).

\section{Array comparative genomic hybridization}

DNA samples from subjects CAR54.03, CAR615.01, CAR696.01, and CAR767.03 were studied by array comparative genomic hybridization (aCGH). Customized high-density (HD) aCGH (Agilent Technology, Santa Clara, CA, USA) in an 8X60K format was designed to interrogate the 19p13.2p13.12 critical region (chr19: 12907 616-15 565 924, GRCh37/hg19). The probe density averaged five oligonucleotides per kilobase for the critical region identified with copy number alterations. The array was also designed to interrogate the flanking genomic regions of up to $6 \mathrm{Mb}$ in size with a probe density of $1-2 / \mathrm{kb}$. Previously described protocols (5) with minor modifications were followed for aCGH's experimental procedures, including DNA fragmentation, labeling and hybridization, array scanning, and data processing. We excluded $19 p$ duplication in the parents of patients 1 and 2, and DNA was not available for patient 3 .

\section{Breakpoint junction analysis}

Breakpoint junction analysis was performed to examine rearrangement product structures and surmise the potential underlying mechanisms for the complex genomic rearrangements observed from the aCGH data. Long-range (LR) PCRs were used to amplify the breakpoint junctions. Forward and reverse primers were designed using the sequences from the estimated copy number variant $(\mathrm{CNV})$ boundaries as determined by changes in copy number states on the aCGH $\log _{2}$ ratio plot. TaKaRa LA Taq (Clontech, \#RR002A) was used for the PCR amplifications. Sanger sequencing was performed for the PCR products, and the DNA sequences were compared with the reference genome (hg19) in order to precisely map the breakpoint junctions.

\section{CNV analyses}

Individual CNV assays were performed by duplex TaqMan real-time PCR assays in order to independently verify the aCGH results in the patients and to extend the analysis in a cohort of patients with sporadic BAH. CNV assays for 
PRKACA utilized a pair of unlabeled primers, and a FAMlabeled MGB probe was supplied from Life Technologies. RNase P (Life Technologies, \#4403328) with a VIC-labeled TAMRA probe was used as a reference gene. TaqMan CNV assays were performed according to the manufacturer's protocol (Life Technologies). Briefly, experiments were prepared in 96-microwell plates and consisted of $20 \mu \mathrm{l}$ reaction volumes containing $20 \mathrm{ng}$ genomic DNA, $10 \mu \mathrm{l}$ TaqMan Genotyping Master Mix (Life Technologies, catalog number 4371355), and $1 \mu \mathrm{l}$ each of one target gene and reference $\mathrm{CNV}$ assay mixes. All reactions were run in triplicate on a ViiA 7 Real-Time PCR System (Life Technologies) and thermal cycling conditions were $95^{\circ} \mathrm{C}$, for 10 min followed by 40 cycles of $95^{\circ} \mathrm{C}$ for $15 \mathrm{~s}$ and $60{ }^{\circ} \mathrm{C}$ for $1 \mathrm{~min}$. All data were analyzed using the CopyCaller Software version 2.0 (Life Technologies). The copy number was determined by the relative relationship between the quantity of the target gene and the reference gene, using a female calibrator sample known to have two copies of each gene as the basis for comparison.

\section{Immunohistochemistry and clinical assays}

The adrenal glands from all patients were processed as per routine histopathological procedures for diagnostic purposes. Hematoxylin and eosin (H\&E)-stained slides and other appropriate immunohistochemical (IHC) stainings (for example, inhibin-A dilution 1:100, Biogenex, Inc. (Fremont, CA, USA) and synaptophysin, dilution 1:100, Zymed, Inc. (Carlsbad, CA, USA)) were reviewed. Detailed analysis of the pathology of these patients has been reported previously (6). IHC on unstained slides from breast tumor tissue for patient CAR615.05 was performed in collaboration with Histoserve, Inc. (Germantown, MD, USA) using standard procedures. The primary antibody used was rabbit anti-Ca, sc903 (Santa Cruz Biotechnology) and the secondary antibody was rabbit polyclonal anti-IgG (Sigma-Aldrich, Inc.). Adrenocorticotropic hormone (ACTH) was measured using the chemiluminescence immunoassay on an Immulite 200 XPi analyzer (Siemens, Malvern, PA, USA). Serum cortisol was measured using the chemiluminescence immunoassay on the Siemens Immulite 2500 analyzer (Siemens).

\section{Results}

\section{Clinical history and manifestations}

Patient 1 (CAR54.03) presented at the age of 8 with a yearlong history of progressive facial swelling, prominent hirsutism on the face and back, decreased linear growth,
$9 \mathrm{~kg}$ weight gain in 1 year, facial plethora, and hypertension. During the preceding 6 months, he complained of intermittent headaches and decreased energy. The patient had no significant past medical history; his maternal grandfather died at the age of 39 due to a myocardial infarction. Physical examination revealed a pigmented left inner canthus, as well as pigmented spots on the vermilion border and facial freckling (Fig. 1). He was hypertensive with systolic blood pressures ranging from 125 to $140 \mathrm{mmHg}$ (normal range (NR) for an eight year old is $>116 / 77)$. An evaluation for hypercortisolemia showed that he failed to suppress to $1 \mathrm{mg}$ overnight dexamethasone, with a post-dexamethasone cortisol level remaining elevated (Table 1); ACTH was undetectable. Further evaluation confirmed hypercortisolemia with elevated urinary free cortisol (UFC), and loss of diurnal cortisol variation with a midnight cortisol of 974.3 (NR: <121 nmol/l) and morning cortisol of $1010.2 \mathrm{nmol} / 1$ (NR: 138-690 nmol/l). Corticotropin-releasing hormone (CRH) stimulation test was flat with a baseline cortisol of $1037.8 \mathrm{nmol} / \mathrm{l}$ and a peak of $1035 \mathrm{nmol} / \mathrm{l}$; ACTH remained suppressed throughout the test. There was a paradoxical increase in UFC levels in response to the Liddle's test (2 days of low-dose dexamethasone $0.5 \mathrm{mg}$ by mouth every $6 \mathrm{~h}$, followed by 2 days of high-dose dexamethasone $2 \mathrm{mg}$ by mouth every $6 \mathrm{~h}$ ). The patient had a baseline UFC of $1821.6 \mathrm{nmol} /$ day, rising to a peak of $2484 \mathrm{nmol} /$ day at the end of high-dose dexamethasone, corresponding to a 73.4\% increase in UFC. A computed tomography (CT) scan revealed bilateral adrenal nodularity with slight thickening and irregularity of both glands with multiple nodules $<5 \mathrm{~mm}$; the pituitary magnetic resonance imaging (MRI) was normal. The patient underwent bilateral adrenalectomy and pathological findings were consistent with a form of BAH characterized by cytomegaly, persistence of the transient cortex, and hyperplasia of the permanent cortex that has been reported previously in other patients with similar clinical presentation (Fig. 2) (7). No sequence change in PRKACA was identified, but combined commercially available aCGH and custom designed HD-aCGH revealed a $2.7 \mathrm{Mb}$ duplication encompassing PRKACA on chromosome 19p13.2p13.12 (4). The patient was screened for manifestations of CNC including thyroid and testicular ultrasounds and echocardiogram; all were normal. He continues to do well 5 years after adrenalectomy.

Patient 2 (CAR696.01) presented at the age of 2 years and 6 months with rapid weight gain and centripetal obesity, hirsutism, moon facies, posterior cervical fat pad, flushed cheeks, muscle weakness, fatigue, and easy irritability. He had mild developmental delay. He was 


\section{CAR54.03}

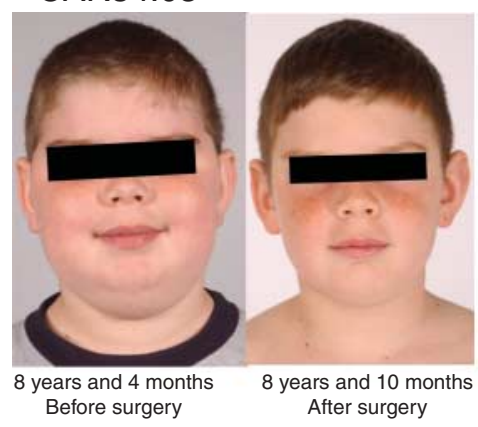

CAR696.01

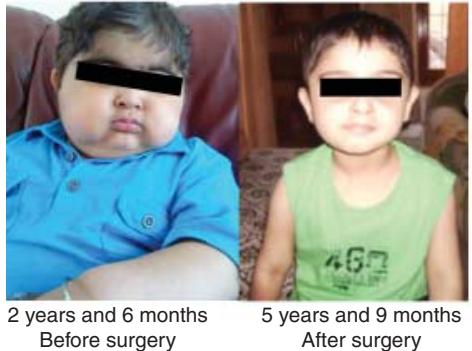

CAR767.03
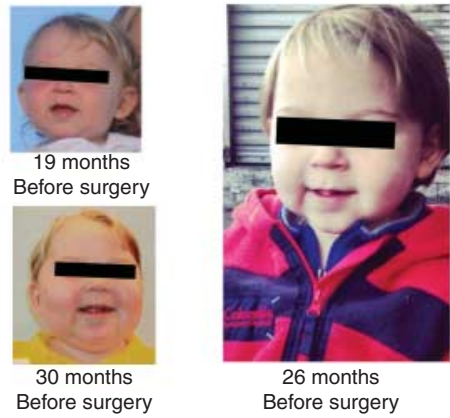

Chr19: 12346258-16058758

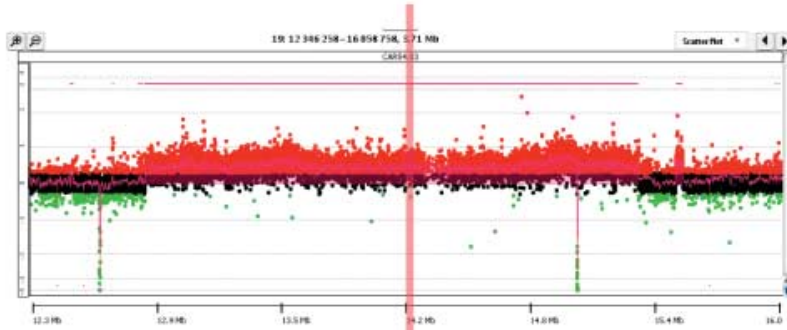

$\log _{2}$ ratio $=0.50$

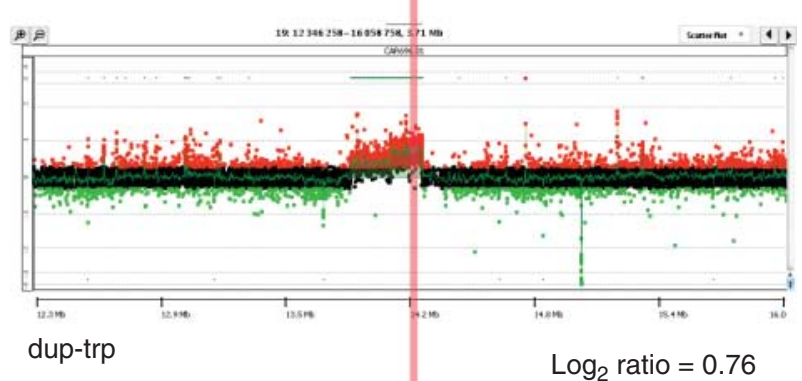

$\log _{2}$ ratio $=0.76$

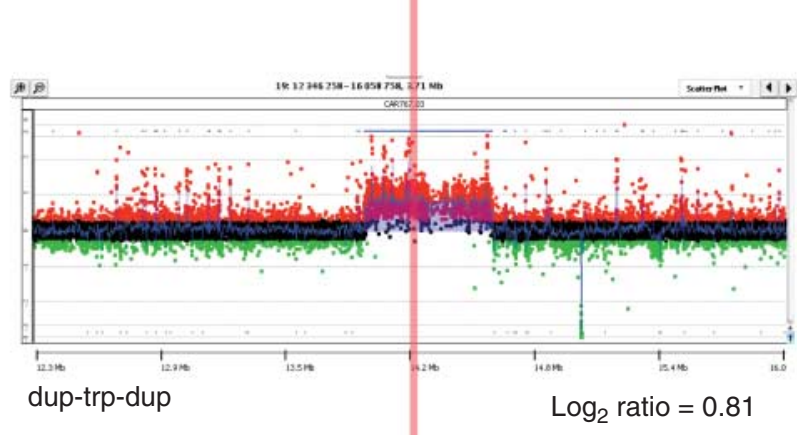

PRKACA

\section{Figure 1}

Facial appearance of patients 1-3 before and after adrenalectomy and their corresponding HD-aCGH results. The photographs of the patients are shown in the left column, and their HD-aCGH studies are shown accordingly in the right column. aCGH plots covering the critical region (chr19: 12346 25816058 758) are shown. Red dots, probes with $\log _{2}$ ratio >0.25;

delivered at 36 weeks gestation and had neonatal hypoglycemia. He had a healthy older brother and no family history of endocrinopathy. On examination, his height was in the $25 \%$ with weight $>99 \%$; no skin lesions consistent with CNC were noted (Fig. 1). Biochemical evaluation confirmed hypercortisolemia with an elevated 24-h UFC (Table 1), and absent diurnal variation (midnight serum cortisol $811.4 \mathrm{nmol} / \mathrm{l}$ and $8-\mathrm{h}$ cortisol black dots, probes with $\log _{2}$ ratio between -0.25 and 0.25 ; and green dots, probes with $\log _{2}$ ratio smaller than -0.25 . The vertical red bar represents the position of PRKACA, and the $\log _{2}$ ratios of the amplified segments encompassing PRKACA are listed under each aCGH plot.

$850.1 \mathrm{nmol} / \mathrm{l})$. ACTH remained undetectable, consistent with an adrenal source of CS. Dexamethasone administration in the Liddle's test ( 2 days of $20 \mu \mathrm{g} / \mathrm{kg}$ per day followed by 2 days of $80 \mu \mathrm{g} / \mathrm{kg}$ per day) did not cause suppression of cortisol. ACTH levels remained low throughout the CRH test, while cortisol levels remained elevated, both consistent with primary adrenal disease. Adrenal CT scan was read as normal. The patient 
Table 1 Patients' baseline characteristics.

\begin{tabular}{|c|c|c|c|c|c|}
\hline $\begin{array}{l}\text { Patient } \\
\text { number }\end{array}$ & Patient code & Sex & $\begin{array}{l}\text { PRKACA } \\
\text { CNV }\end{array}$ & $\begin{array}{c}\text { Age at } \\
\text { diagnosis } \\
\text { (years) }\end{array}$ & $\begin{array}{c}\text { ACTH } \\
(\mathrm{pmol} / \mathrm{l})\end{array}$ \\
\hline 1 & CAR54.03 & Male & dup & 8 & $<1.10$ \\
\hline 2 & CAR696.01 & Male & $\operatorname{trp}$ & 2.5 & $<1.10$ \\
\hline 3 & CAR767.03 & Male & $\operatorname{trp}$ & 2.6 & 1.58 \\
\hline 4 & CAR615.05 & Female & dup & 39 & $<2.20$ \\
\hline 5 & CAR615.01 & Male & dup & 23 & $<1.10$ \\
\hline
\end{tabular}

\begin{tabular}{|c|c|}
\hline $\begin{array}{c}\text { Midnight } \\
\text { salivary cortisol } \\
(\mathrm{nmol} / \mathrm{l})\end{array}$ & $\begin{array}{c}\text { Midnight } \\
\text { serum cortisol } \\
(\mathrm{nmol} / \mathrm{l})\end{array}$ \\
\hline & $\begin{array}{l}974.3 \\
811.4\end{array}$ \\
\hline 43.33 & \\
\hline
\end{tabular}

\begin{tabular}{|c|c|}
\hline $\begin{array}{l}\text { Serum cortisol } \\
\text { after Dex } \\
(\mathrm{nmol} / \mathrm{l})\end{array}$ & $\begin{array}{c}\text { 24-h urinary } \\
\text { cortisol } \\
\text { (nmol/day) }\end{array}$ \\
\hline 786.6 & $1322-2221.8$ \\
\hline 651.4 & 703.8 \\
\hline 604.4 & \\
\hline 648.6 & 568.6 \\
\hline 772.8 & \\
\hline
\end{tabular}

dup, duplication; trp, triplication.

underwent bilateral adrenalectomy and pathological findings were diffuse cortical hyperplasia and prominent capsular and extra-capsular micronodular cortical hyperplasia, consistent with iMAD (6). No sequence change in PRKACA was identified, but, on a commercially available aCGH, a $294 \mathrm{~kb}$ gain at chromosome 19p13.13p13.12 was found (4). Follow-up HD-aCGH study revealed a complex genomic gain of distal duplication followed by a proximal triplication (dup-trp); PRKACA was included in the triplicated segment (see also below and Supplementary Figure 1, see section on supplementary data given at the end of this article). Most recently, at the age of 5 years and 9 months, his height had improved to the 75th percentile and there were no signs of CNC or any other conditions.

Patient 3 (CAR767.03) presented at the age of 2 years and 7 months with a rapid weight gain of $8 \mathrm{~kg}$ over 6 months, and linear growth deceleration and the corresponding cross in height from the 75th to the 5th percentile. The family had noted a dramatic change in his appearance and behavior over the preceding 6 months (Fig. 1). The patient was large at birth and had transient hypoglycemia and macroglossia requiring surgical intervention at the age of 2; he was investigated for possible Beckwith-Wiedemann syndrome, but he had no other signs of this condition. The patient has three healthy siblings. On examination, he had classic stigmata of CS and was hypertensive. Biochemical evaluation confirmed hypercortisolism; midnight salivary free cortisol level was elevated to $43.33 \mathrm{nmol} / 1$ (normal <2.76). Morning cortisol remained elevated following low-dose dexamethasone (0.5 mg) suppression testing (at $604.4 \mathrm{nmol} / \mathrm{l})$. ACTH was low or undetectable. Adrenal imaging revealed bilateral nodular hyperplasia with multiple nodules $<5 \mathrm{~mm}$. The patient underwent bilateral adrenalectomy. Pathological diagnosis was consistent with cortical hyperplasia, and prominent capsular and extra-capsular micronodular cortical hyperplasia consistent with a mixed pathology of iMAD and/or PMAH (6). At 1-year follow-up, he was normotensive and had marked improvement in linear growth and normal appetite, with weight decreasing to the $50 \%$ and height back up to the $25 \%$. No sequence change in PRKACA was identified, but a commercially available aCGH showed a $551 \mathrm{~kb}$ gain on chromosome 19p13.13p13.12 (4). Follow-up HD-aCGH study revealed a complex genomic gain of a pattern of a distal duplication followed by a proximal triplication subsequently followed by another proximal duplication (dup-trp-dup) with PRKACA residing within the triplicated segment (Supplementary Figure 1); the rearrangement was complex (see below) and similar to what has been observed in other genomic disorders (8).

Patients 4 and 5 are a mother and son who presented at different times with CS. Patient 4 (CAR615.05) was

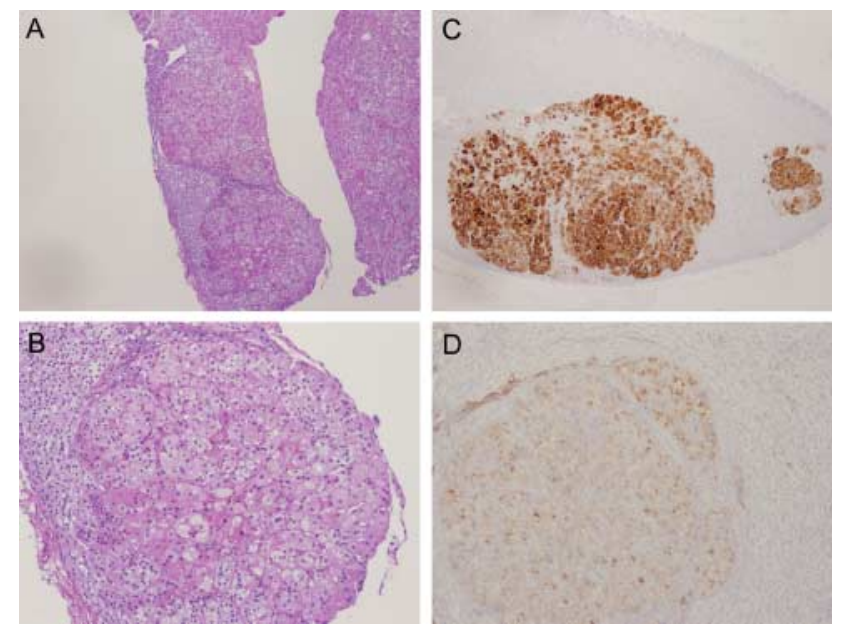

\section{Figure 2}

Histopathology from adrenal glands in patient 1. (A and B) Histological sections show an adrenal gland with cortical nodular hyperplasia. Multiple nodules are present, which contain compact and few lipid-rich cells (A, H\&E $40 \times$ original magnification and $B, H \& E 100 \times$ original magnification). (C and D) Hyperplastic nodules are highlighted by immunohistochemistry stains for synaptophysin (C, $100 \times$ original magnification) and inhibin (D, $40 \times$ original magnification). 
a 39-year-old woman who presented with hypertension, weight gain, hirsutism, bruising, depression, irritability, moon facies, buffalo hump, and headaches. Biochemical evaluation revealed hypercortisolism with an elevated UFC with low ACTH levels (Table 1). The serum cortisol did not suppress to high-dose $(8 \mathrm{mg})$ dexamethasone (Table 1). Adrenal CT revealed an enlarged left adrenal, $1.2 \mathrm{~cm}$ in maximum diameter, and a normal appearing right adrenal. Following left adrenalectomy, the CS remitted, but recurred at the age of 43 , prompting a right adrenalectomy. Pathological examination of both adrenal glands was consistent with macronodular hyperplasia (PMAH). The patient developed a well-differentiated infiltrating tubular breast carcinoma, $4 \mathrm{~mm}$ in size, and extensive ductal carcinoma in situ at the age of 49 years; she is currently well. Genetic testing showed no sequence changes in known genes, but a commercially available aCGH/chromosomal microarray analysis revealed a $616 \mathrm{~kb}$ gain on chromosome 19p13.2 (4). Subsequent IHC of the patient's breast carcinoma for the PRKACA subunit was positive (Supplementary Figure 2, see section on supplementary data given at the end of this article).

Patient 5 (CAR615.01) is the son of patient 4; he presented at the age of 23 years with avascular necrosis of the left femoral head and hypertension. CS was suspected; biochemical analysis confirmed hypercortisolism with elevated cortisol after a high-dose $(8 \mathrm{mg})$ dexamethasone suppression test (Table 1). Baseline ACTH was undetectable; adrenal MRI with contrast showed an enhancing left adrenal ovoid mass measuring $2.6 \times 2.4 \mathrm{~cm}$ with an irregular periphery. The patient underwent left adrenalectomy; pathological findings were consistent with PMAH. At the age of $29, \mathrm{BMI}$ was $27.6 \mathrm{~kg} / \mathrm{m}^{2}$, there was hypertension, and borderline elevation of morning cortisol following a $1 \mathrm{mg}$ overnight dexamethasone suppression test, but UFC was normal (Table 1). He had the maternally inherited $616 \mathrm{~kb}$ gain on $19 \mathrm{p} 13.2$ by the commercially available aCGH analysis (4).

\section{Genomic gains revealed by HD-aCGH}

The critical region with genomic gains shared, among the subjects, including CAR54.03, CAR615.01, CAR696.01, and CAR767.03, was identified previously by genomewide aCGH (4). Customized HD-aCGH interrogating the critical region delineated the genomic gains in the DNA samples from these four families, and revealed complex genomic gains based on the HD-aCGH data: a distal duplicated segment followed by a normal segment subsequently followed by a proximal duplicated segment (dup-nml-dup) was observed in CAR54.03; a distal duplication followed by a proximal triplication (dup-trp) was observed in CAR696.01; and a distal duplication followed by a proximal triplication subsequently followed by another proximal duplication (dup-trp-dup) was observed in CAR767.03 (Fig. 1). The genomic gain in subject CAR615.01 was barely visible due to the low quality of the aCGH result, which might result from the low quality of the DNA sample from this subject (data not shown). Therefore, details of the gain in the genome of CAR615.01 could not be fully ascertained.

All the genomic gains observed in the four individuals were complex genomic rearrangements (CGRs) with variable breakpoint junctions, and these gains encompassed the gene PRKACA. The PRKACA gene resided on duplication segments in subject CAR54.03, while it was triplicated in subjects CAR696.01 and CAR767.03. Although a chromosomal gain could be observed based on the aCGH data of subject CAR615.01, the exact copy number state of the PRKACA gene could not be estimated.

\section{Breakpoint junction analyses of the genomic gains}

LR-PCR and Sanger sequencing were attempted for the breakpoint junction (Jct) analyses of all the genomic gains. In the 'dup-nml-dup' observed in CAR54.03, at least two breakpoint junctions (Jct1 and Jct2) are expected to be involved in the rearrangement. Breakpoint analyses revealed Jct1, which connected the proximal end of the distal duplicated segment and the distal end of the proximal duplicated segment. A 2 bp microhomology (GG) was observed at the breakpoint junctions, suggesting the Fork Stalling and Template Switching/MicrohomologyMediated Break-Induced Replication (FoSTeS/MMBIR) as the potential mechanism for the formation of Jct1 $(9,10,11)$. Attempts to map the other breakpoint(s) in the 'dupnml-dup' were unsuccessful. The breakpoint mapping for the 'dup-trp' rearrangement in CAR696.01 was also not successful. These results might suggest potential invisible complexity underlying the rearrangement that generated the 'dup-nml-dup' and 'dup-trp' complex structure observed in subjects CAR54.03 and CAR696.01. Regarding the 'dup-trp-dup' observed in CAR767.03, two breakpoint junction clusters were revealed by breakpoint analysis (Jct1 and Jct2). Two Alu repetitive elements, AluSx3 and AluSx1, were found at the Jct1. These two Alus, sharing $73.7 \%$ identity with direct orientation, might serve as substrates for $A l u-A l u$ mediated recombinational rearrangement (8). A 6 bp microhomology (TACAGG) was identified at the Jct1, which was flanked by highly 
identical sequences. In Jct2, a 3 bp insertion (ACA) was identified at the junction. It might be explained by the FoSTeS/MMBIR mechanism by two potential template switches to generate the insertion.

The Jct1 observed in the 'dup-nml-dup' of subject CAR54.03 might be part of the CGR, or it might be a breakpoint junction of a deletion of a genomic segment on the homologous chromosome. However, the parental DNA was not available for testing the actual mechanism. According to the Jct1 and Jct2 observed in the 'dup-trpdup' of subject CAR767.03, the gains were likely to result from CGRs: the two Alus (AluSx 1 and AluSx3) aligned at the Jct1 indicated an Alu-mediated recombination, while the observance of the $3 \mathrm{bp}$ insertion (ACA) at the Jct2 favored the FoSTeS/MMBIR as the mechanism.

\section{Discussion}

The five patients had CS in association with a germline copy number gain resulting in amplification of the PRKACA gene encoding the most important catalytic subunit of the cAMP-dependent protein kinase or PKA. They are the first reported patients with a CNV involving this locus and implicated in a specific phenotype. As we have reported previously $(4,6)$, it appears that germline amplification of PRKACA leads to BAH, both macronodular and micronodular forms. In this study, we reexamined the genetics and genomics of these patients with in-depth genomic analysis and found that there were differences in the degree of amplification: PRKACA triplications were identified in patients 2 and 3, both of whom had clinical presentation at a younger age, when compared with the other patients. However, this observation needs to be repeated in a larger sample size before definitive conclusions are drawn. A similar genomic dosage-dependent phenotype has been described in Charcot-Marie-Tooth disease type 1A (CMT1A): in CMT1A, patients with triplications presented at an earlier age with a more severe distal symmetric polyneuropathy phenotype than those with duplications (12).

These data also demonstrate that amplifications of PRKACA at the genomic level, at least up to triplications, are compatible with life (13). No PRKACA-activating mutations associated with CS have been identified in the germline $(4,13)$, whereas such mutations have now been confirmed in the somatic state by five more studies of hundreds of patients with adrenal CS $(14,15,16,17,18)$. Similarly, in the only cancer where PRKACA is reported to be involved, its activation is also somatic and is caused by genomic rearrangements of the $19 \mathrm{p} 13$ locus resulting in recurrent DNAJB1-PRKACA chimeric transcripts found in fibrolamellar hepatocellular carcinoma (19).

The somatic PRKACA mutations identified to date $(4,14,15,16,17,18)$ are all present in a location of the $\mathrm{C} \alpha$ protein that is responsible for binding to the regulatory subunit(s). They, as a result, lead to activation of PKA by making the catalytic subunit of the enzyme largely inaccessible to its main controller and binding partner, the PKA regulatory subunit(s) $(20,21)$. The cAMPdependent protein kinase or PKA is a tetramer that consists of two inactive catalytic subunits and two regulatory subunits $(20,22)$. The major regulatory subunit of PKA is PRKAR1A, the gene responsible for CNC (there are three other regulatory subunits, PRKAR1B, PRKAR2A, and PRKA2B), and the major catalytic subunit of PKA is PRKACA (there are three other catalytic subunits, PRKACB, PRKACG, and PRKX); in the inactive tetramer, each molecule of $\mathrm{C} \alpha$ is bound by a regulatory subunit (3). The two heterodimers are mainly held together by the two inactivating regulatory subunits; when two molecules of cAMP bind to each regulatory subunit, the tetramer dissolves and the catalytic subunits are free to phosphorylate downstream targets $(3,22)$. Thus, the only controller of the catalytic subunit's activity is its interaction with its binding partner, the regulatory subunit $(3,20,22)$. It should be noted that PRKACA mutations that were predicted to lose interaction with the regulatory subunit while maintaining enzymatic activity had been studied in vitro in the early 1990 s $(23,24,25)$. Additional PRKACA sequences that had similar effects in vitro have been described recently, and the first PRKACA mutations in humans with predicted functional effects were reported in 2012 with no association with an obvious phenotype (26). The first study to investigate the presence of PRKACA mutations in endocrine lesions failed to show any defects in a large collection of thyroid and pituitary adenomas (27). More recently, 92 specimens from sporadic somatotrope adenomas were screened for mutations of PRKACA or $P R K A C B$, and none were identified (28).

The data presented here and those reported earlier $(4,14,15,16,17,18)$ show that PRKACA is a relatively unique endocrine tumor gene: it causes rare forms of $\mathrm{BAH}$ leading to CS in children and young adults when it is part of increased dosage of chromosome 19p13.2p13.12 genomic material; triplications appear to convey a more severe phenotype than duplications. It then causes the relatively common CPA leading to CS in older age when it acquires mutations in the somatic state within the adrenal cortex of one gland. The Leu206Arg and the other activating PRKACA mutations identified to date $(4,14,15,16,17,18)$ may not 
be compatible with life in human embryos and that is potentially why they have not been found in the germline.

In conclusion, dosage of PKA catalytic activity appears to determine the phenotype of the $\mathrm{CS}$ in humans (i.e., $\mathrm{BAH}$ vs CPA) (20). Functional studies in fruit flies suggested a dosage-dependent effect of PKA activity on the phenotype (29). This would be expected from a protein with its activity tightly controlled by cAMP and the PKA regulatory subunits $(3,20,22)$. A complete knockout of the Prkaca gene is not compatible with life in most mice; few animals in certain backgrounds survive birth but die later in life (30); the haploinsufficient mouse has a few abnormalities such as mildly decreased bone mineral density (31). Another question has a less clear answer for a ubiquitously present molecule such as PRKACA: why is only the adrenal cortex affected in patients with germline defects? The adrenal cortex may simply be exquisitely sensitive to PRKACA dosage and/or activity, a hypothesis that requires testing in animal models, as we have suggested previously (20). Indeed, new animal models that will study Prkaca overexpression are essential to elucidate the pathophysiology of the described PRKACA defects and will be very useful for the development of molecular therapies for the treatment of cortisol-producing lesions in humans.

\section{Supplementary data}

This is linked to the online version of the paper at http://dx.doi.org/10.1530/ EJE-14-1154.

\section{Declaration of interest}

The authors declare that there is no conflict of interest that could be perceived as prejudicing the impartiality of the research reported.

\section{Funding}

This research was supported by the Intramural division of the Eunice Kennedy Shriver National Institute of Child Health and Human Development, and National Institutes of Health and extramural grants NINDS RO1NS058529 and NHGRI U54HG006542. Clinical trial registration number: NCT00001452.

\section{Acknowledgements}

The authors thank Mr Jeremy Swan (NICHD, NIH) for help with preparation of the figures. Finally, they thank the patients and their families for participating in this research.

\section{References}

1 Kirschner LS, Carney JA, Pack SD, Taymans SE, Giatzakis C, Cho YS, Cho-Chung YS \& Stratakis CA. Mutations of the gene encoding the protein kinase A type I- $\alpha$ regulatory subunit in patients with the Carney complex. Nature Genetics 200026 89-92. (doi:10.1038/79238)

2 Bertherat J, Groussin L, Sandrini F, Matyakhina L, Bei T, Stergiopoulos S, Papageorgiou T, Bourdeau I, Kirschner LS, Vincent-Dejean C et al. Molecular and functional analysis of PRKAR1A and its locus (17q22-24) in sporadic adrenocortical tumors: $17 \mathrm{q}$ losses, somatic mutations, and protein kinase A expression and activity. Cancer Research 200363 5308-5319.

3 Meoli E, Bossis I, Cazabat L, Mavrakis M, Horvath A, Stergiopoulos S, Shiferaw ML, Fumey G, Perlemoine K, Muchow M et al. Protein kinase A effects of an expressed PRKAR1A mutation associated with aggressive tumors. Cancer Research 200868 3133-3141. (doi:10.1158/0008-5472. CAN-08-0064)

4 Beuschlein F, Fassnacht M, Assie G, Calebiro D, Stratakis CA, Osswald A, Ronchi CL, Wieland T, Sbiera S, Faucz FR et al. Constitutive activation of PKA catalytic subunit in adrenal Cushing's syndrome. New England Journal of Medicine 2014370 1019-1028. (doi:10.1056/ NEJMoa1310359)

5 Shinawi M, Liu P, Kang SH, Shen J, Belmont JW, Scott DA, Probst FJ, Craigen WJ, Graham BH, Pursley A et al. Recurrent reciprocal 16p11.2 rearrangements associated with global developmental delay, behavioural problems, dysmorphism, epilepsy, and abnormal head size. Journal of Medical Genetics 201047 332-341. (doi:10.1136/jmg.2009. 073015)

6 Carney JA, Lyssikatos C, Lodish MB \& Stratakis CA. Germline PRKACA amplification leads to Cushing syndrome caused by 3 adrenocortical pathologic phenotypes. Human Pathology 201546 40-49.

7 Carney JA, Ho J, Kitsuda K, Young WF Jr \& Stratakis CA. Massive neonatal adrenal enlargement due to cytomegaly, persistence of the transient cortex, and hyperplasia of the permanent cortex: findings in Cushing syndrome associated with hemihypertrophy. American Journal of Surgical Pathology 201236 1452-1463. (doi:10.1097/PAS. Ob013e31825d538b)

8 Carvalho CM, Ramocki MB, Pehlivan D, Franco LM, GonzagaJauregui C, Fang P, McCall A, Pivnick EK, Hines-Dowell S \& Seaver LH. Inverted genomic segments and complex triplication rearrangements are mediated by inverted repeats in the human genome. Nature Genetics 201143 1074-1081.

9 Lee JA, Carvalho CM \& Lupski JR. A DNA replication mechanism for generating nonrecurrent rearrangements associated with genomic disorders. Cell 2007131 1235-1247. (doi:10.1016/j.cell.2007.11.037)

10 Zhang F, Khajavi M, Connolly AM, Towne CF, Batish SD \& Lupski JR. The DNA replication FoSTeS/MMBIR mechanism can generate genomic, genic and exonic complex rearrangements in humans. Nature Genetics 200941 849-853. (doi:10.1038/ng.399)

11 Hastings PJ, Ira G \& Lupski JR. A microhomology-mediated break-induced replication model for the origin of human copy number variation. PLoS Genetics 20095 e1000327.

12 Liu P, Gelowani V, Zhang F, Drory VE, Ben-Shachar S, Roney E, Medeiros AC, Moore RJ, DiVincenzo C, Burnette WB et al. Mechanism, prevalence, and more severe neuropathy phenotype of the CharcotMarie-Tooth type 1A triplication. American Journal of Human Genetics 201494 462-469. (doi:10.1016/j.ajhg.2014.01.017)

13 Stratakis CA. E pluribus unum? The main protein kinase A catalytic subunit (PRKACA), a likely oncogene, and cortisol-producing tumors Journal of Clinical Endocrinology and Metabolism 201499 3629-3633. (doi:10.1210/jc.2014-3295)

14 Sato Y, Maekawa S, Ishii R, Sanada M, Morikawa T, Shiraishi Y, Yoshida K, Nagata Y, Sato-Otsubo A, Yoshizato T et al. Recurrent somatic mutations underlie corticotropin-independent Cushing's syndrome. Science 2014344 917-920. (doi:10.1126/science.1252328)

15 Goh G, Scholl UI, Healy JM, Choi M, Prasad ML, Nelson-Williams C, Kunstman JW, Korah R, Suttorp AC, Dietrich D et al. Recurrent activating mutation in PRKACA in cortisol-producing adrenal tumors. Nature Genetics 201446 613-617. (doi:10.1038/ng.2956) 
16 Cao Y, He M, Gao Z, Peng Y, Li Y, Li L, Zhou W, Li X, Zhong X, Lei Y et al. Activating hotspot L205R mutation in PRKACA and adrenal Cushing's syndrome. Science 2014344 913-917. (doi:10.1126/science. 1249480)

17 Di Dalmazi G, Kisker C, Calebiro D, Mannelli M, Canu L, Arnaldi G, Quinkler M, Rayes N, Tabarin A, Laure Jullie M et al. Novel somatic mutations in the catalytic subunit of the protein kinase A as a cause of adrenal Cushing's syndrome: a European multicentric study. Journal of Clinical Endocrinology and Metabolism 201499 E2093-E2100. (doi:10.1210/jc.2014-2152)

18 Nakajima Y, Okamura T, Gohko T, Satoh T, Hashimoto K, Shibusawa N, Ozawa A, Ishii S, Tomaru T, Horiguchi K et al. Somatic mutations of the catalytic subunit of cyclic AMP-dependent protein kinase (PRKACA) gene in Japanese patients with several adrenal adenomas secreting cortisol [Rapid Communication]. Endocrine Journal 201461 825-832. (doi:10.1507/endocrj.EJ14-0282)

19 Honeyman JN, Simon EP, Robine N, Chiaroni-Clarke R, Darcy DG, Lim II, Gleason CE, Murphy JM, Rosenberg BR, Teegan L et al. Detection of a recurrent DNAJB1-PRKACA chimeric transcript in fibrolamellar hepatocellular carcinoma. Science 2014343 1010-1014. (doi:10.1126/ science.1249484)

20 Stratakis CA. E pluribus unum? The main protein kinase A catalytic subunit (PRKACA), a likely oncogene, and cortisol-producing tumors. Journal of Clinical Endocrinology and Metabolism 201499 3629-3633. (doi:10.1210/jc.2014-3295)

21 Kirschner LS. Medicine. A unified cause for adrenal Cushing's syndrome. Science 2014344 804-805. (doi:10.1126/science.1254901)

22 Stratakis CA. cAMP/PKA signaling defects in tumors: genetics and tissue-specific pluripotential cell-derived lesions in human and mouse. Molecular and Cellular Endocrinology 2013371 208-220. (doi:10.1016/ j.mce.2013.01.015)

23 Orellana SA, Amieux PS, Zhao X \& McKnight GS. Mutations in the catalytic subunit of the cAMP-dependent protein kinase interfere with holoenzyme formation without disrupting inhibition by protein kinase inhibitor. Journal of Biological Chemistry 1993268 6843-6846.

24 Orellana SA \& McKnight GS. The S49 Kin- cell line transcribes and translates a functional mRNA coding for the catalytic subunit of cAMP-dependent protein kinase. Journal of Biological Chemistry 1990 265 3048-3053.

25 Orellana SA \& McKnight GS. Mutations in the catalytic subunit of cAMP-dependent protein kinase result in unregulated biological activity. PNAS 199289 4726-4730. (doi:10.1073/pnas.89.10.4726)

26 Soberg K, Larsen AC, Diskar M, Backe PH, Bjoras M, Jahnsen T, Laerdahl JK, Rognes T, Herberg FW \& Skalhegg BS. Identification and characterization of novel mutations in the human gene encoding the catalytic subunit C $\alpha$ of protein kinase A (PKA). PLoS ONE 20127 e34838. (doi:10.1371/journal.pone.0034838)

27 Esapa CT \& Harris PE. Mutation analysis of protein kinase A catalytic subunit in thyroid adenomas and pituitary tumours. European Journal of Endocrinology 1999141 409-412. (doi:10.1530/eje.0.1410409)

28 Larkin SJ, Ferrau F, Karavitaki N, Hernandez-Ramirez LC, Ansorge O, Grossman AB \& Korbonits M. Sequence analysis of the catalytic subunit of PKA in somatotroph adenomas. European Journal of Endocrinology 2014171 705-710. (doi:10.1530/EJE-14-0545)

29 Collier LS, Suyama K, Anderson JH \& Scott MP. Drosophila Costal1 mutations are alleles of protein kinase A that modulate hedgehog signaling. Genetics 2004167 783-796. (doi:10.1534/genetics.103. 024992)

30 Skalhegg BS, Huang Y, Su T, Idzerda RL, McKnight GS \& Burton KA. Mutation of the C $\alpha$ subunit of PKA leads to growth retardation and sperm dysfunction. Molecular Endocrinology 200216 630-639.

31 Tsang KM, Starost MF, Nesterova M, Boikos SA, Watkins T, Almeida MQ, Harran M, Li A, Collins MT, Cheadle C et al. Alternate protein kinase A activity identifies a unique population of stromal cells in adult bone. PNAS 2010107 8683-8688. (doi:10.1073/pnas. 1003680107)

Received 29 December 2014

Revised version received 11 March 2015

Accepted 23 March 2015 